\title{
Odontogenic Keratocyst Associated With Supernumerary Tooth: A Case Report
}

\author{
Kumar PD', Devadiga $\mathrm{S}^{2}$, Agrawal $\mathrm{R}^{3}$, Mahabaleshwar $\mathrm{CH}^{4}$
}

\begin{abstract}
The odontogenic keratocyst $(\mathrm{OKC})$ is a cystic lesion of odontogenic origin that demonstrates the behavioral characteristics of a benign neoplasm and has a propensity to recur after surgical treatment. This is a case report a 45-year-old male presented with a solitary swelling in the submandibular region, which was clinically diagnosed as dentigerous cyst. The microscopic examination revealed the presence of odontogenic keratocyst.
\end{abstract}

Key words: Odontogenic keratocyst, Dentigerous cyst.

${ }^{1}$ Professor,

Oral and Maxillofacial Surgery,

Kvg Dental College and Hospital,

Sullia

2 Senior Lecturer,

Oral and Maxillofacial Surgery,

KVG Dental College and Hospital,

Sullia.

${ }^{3} \mathrm{PG}$ Student,

Oral and Maxillofacial Surgery,

KVG Dental College and Hospital,

Sullia.

${ }^{4}$ Professor,

Oral and Maxillofacial Surgery,

KVG Dental College and Hospital,

Sullia

\section{Contact Author}

Dr Romit agrawal

romit_silver1216@yahoo.in

J Oral Health Comm Dent 2015;9(2)96-98

\section{INTRODUCTION}

7 he term "keratocyst" was given by Philipsen in 1956 based on the histologic appearance of its cystic lining (1). Formely it is called as Keratocystic odontogenic tumor because of its aggressive behavior, recurrence rate and genetic abnormalities.OKC (Odontogenickeratocyst) arise from primordial Odontogenic Epithelium like dental lamina or its remnants. The odontogenic keratocyst has been recognized as an aggressive lesion with a propensity for recurrence unless removed completely surgically. They account for 4 to $12 \%$ of all Odontogenic cysts. They have a pronounced peak frequency in the second and third decades, ranging from $40 \%$ to $60 \%$ of patients in this age group (2). They can occur anywhere in the jaws, includingthe midline of the mandible and maxilla. The most common site of occurrence being at the angle of the mandible and can extend for varying distances into the body and ascending ramus (2).

\section{CASE REPORT}

A 45year-old male presented to the Department of Oral and Maxillofacial
Surgery with the chief complaint of a swelling of the left side of lower jaw region since 4 months. On examination patient had a solitary oval swelling measuring approximately $4 \mathrm{X} 4 \mathrm{~cm}$ extending antero posteriorly from left commissure of mouth to $5 \mathrm{~cm}$ anterior to angle of mandible, supero inferiorly line joining corner of ear lobe to lower border of mandible. Over lining skin was smooth. On palpation the swelling was firm to hard in consistency, tender

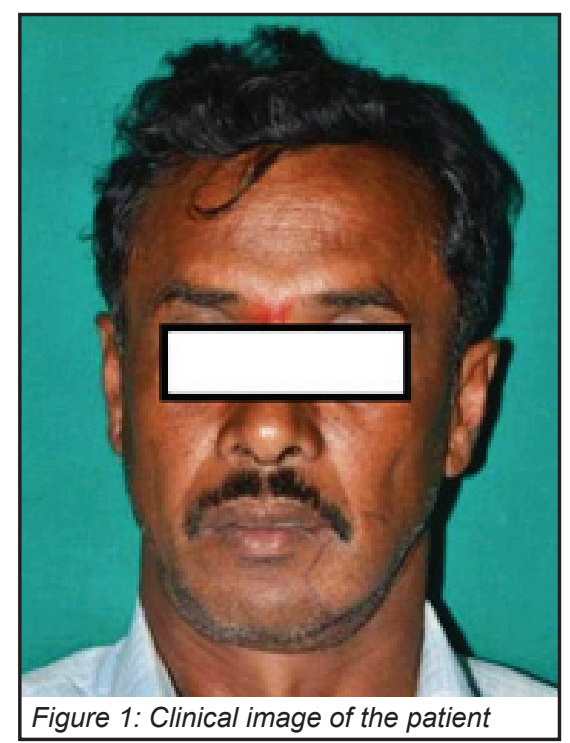




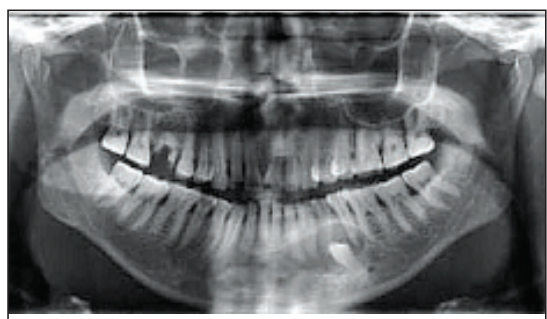

Figure 2: Orthopantamograph showing the lesion

and noncompressible. Intraorally erythematous and obliterated left vestibular sulcus was seen in relation to left canine, first and second premolar. A provisional diagnosis of dentoalveolar abscess was made (Figure 1).

Orthopantamogram showed well defined hazy radiolucency in relation to impacted supernumerary tooth (Figure 2).

Diagnostic aspiration was performed and about $2 \mathrm{ml}$ of reddish brown fluid was aspirated. Biochemistry test performed, and total protein count of 6.2 was found. On the basis of the clinical and radiographic findings, the final diagnosis of dentigerous cyst was made. Intentional root canal treatment of left canine, first and second premolar was done. The patient underwent surgery under general anesthesia an intraoral incision was given. Sub periosteal dissection was made. Cyst enucleation was done along extraction of the supernumerary tooth (Figure 3). Carnoy's solution was applied within the wound defect and removed after 5 minutes. Wound was sutured with 3-o vicryl suture material. The specimen was



then submitted for histopathological examination.

Histopathological sections showed large cystic cavity lined by stratified squamous epithelium with uniform thickness of cells with parakeratin surface and palisaded and polarized basal cells. Supporting connective tissue consists of both loosely arranged and densely packed collagen fibers with both spindle and stellate shaped fibroblasts. Juxtaepithelially there is dense infiltration of chronic inflammatory cells both lymphocytes and plasma cells, numerous blood capillaries filled with RBC's and extravasated RBC's. At the site of inflammation in few areas, epithelium is showing proliferation with thickness more than 10 cells without parakeratin surface. Few satellite cysts are also observed in the connective tissue wall (Figure 4). Histopathological examination showed presence of infected odontogenic keratocyst.

Healing was uneventful and 2months follow up showed no signs of recurrence.

\section{DISCUSSION}

Odontogenic keratocyst (OKC) was first described by Philipsen in 1956, who used this term to describe jaw cysts exhibiting keratinization of their epithelial linings (3). In 2005, the odontogenic keratocyst was renamed by world healthorganization as the keratocystic odontogenic tumor(KOT or KCOT) (4).

In comparison to other cysts of the jaws, the odontogenic keratocyst

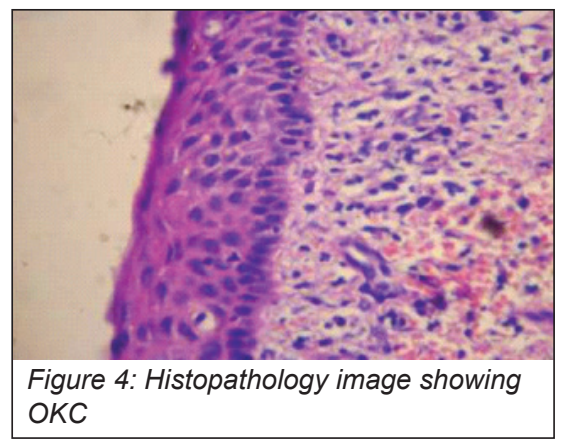

(OKC) is unusual as it shows characteristic clinical features, high recurrence rate and an association with the nevoid basal cell carcinoma syndrome. The distinct histologic feature-the presence of parakeratin, isunique among the inflammatory and developmental cysts that occur in the jaws $(5,6)$.

Although a considerable preference for the mandibular 3rd molar area and ascending ramus exists, OKCs can also occur in the dentate area of maxilla and mandible, presenting themselves as ordinary odontogenic cysts (6). According to Mervyn Shear "The incidence of keratocysts was higher in men than women, that they were considerably more common in whites than in blacks (2).

Typical radiographic features such as scalloped margins,multilobular and multilocular appearance are certainly indicative but are not clear proof of OKCs because other lesions can also exhibit similar radiographical signs (6). An important radiographic and clinical sign is the presence of buccal and lingual cortical plate expansion. OKCs show little tendency to expan$\operatorname{sion}(7,8)$.

Patients with keratocysts may complain of pain, swelling, or discharge. Sometimes they experience paresthesia of the lower lip or teeth. Others are unaware of the lesions until they develop pathologic fractures (8).

Specific features or characteristics of the OKC's behavior are its rate of recurrence when inadequately treated, its tendency to recur in certain surgical sites, and the apparent intrinsic differences between parakeratinized and orthokeratinized variants (2)." The conventional options include (i) enucleation and curettage, (ii) enucleation and peripheral ostectomy, and (iii) osseous resection without (rim ostectomy/ marginal resection) or with (segmental resection) continuity defect (9). 
The controversial chemical agent in use to increase the cure rate of the KCOT is carnoy solution (10). Cryosurgery can also be used to along with enucleation in treating OKCs. Cryosurgey is used as an adjunct along with enucleation and all the principles of enucleation should be used properly (10). According to M.A Pogrel"With simple enucleation, it seems that the recurrence rate may be from $25 \%$ to $60 \%$. When treating the lesion as one would an ameloblastoma, including resection with $1-\mathrm{cm}$ margins (this often necessitates a segmental resection), the recurrence rate can be virtually zero.

We presented a case of infected odontogenic keratocyst in male patient, which was treated with surgical enuceation. Thorough clinical and radiological examination is required for proper treatment. A correct diagnosis is ensured only by histopathological examination of the surgical specimen.

\section{CONCLUSION}

There is clinical, histologic, and genetic evidence that OKCs are benign cystic tumors of odontogenic origin. Myriad surgical strategies have been proposed to facilitate complete removal of the lesion, but the optimum treatment modality has yet to be realized. Recurrence continues to be a common occurrence and should be anticipated when appropriately conservative modalities are used.

\section{REFRENCES:}

1. MalikNA. Textbook of Oral and Maxillofacial surgery 2005; 1stEdition, Jaypee brothers,Noida,401-430.

2. Shear M. Odontogenic keratocysts: clinical features. Oral Maxillofacial Surg Clin N Am 2003;15:335-45.

3. Kolokythas A, Fernandes RP, Pazoki A, Ord RA. OdotogenicKeratocyst: To decompress or not to decompress? a comparative study of decompression and enucleation versus resection/peripheral osteotomy. J Oral And Maxillofacial Surgery 2007;65:640-44.

4. Pogrel MA. The keratocystic odontogenic tumor. Oral And Maxillofacial Surg Clin N Am 2013;25: 21-30.

5. Richard CK. Jordan. Histology and ultrastructural features of the Odontogenic keratocyst. Oral Maxillofacial Surg Clin N Am 2003;15:325-33.

6. Stoelinga PJW. Long-term follow-up on keratocysts treated according to a defined protocol. Int J Oral And Maxillofac Surg 2001;30:14-25.

7. Chapelle Karen AOM, Stoelinga Paul JW, Wilde Peter CM De, Brouns John JA, Voorsmit Ralph ACA. Rationale approach to diagnosis and treatment of ameloblastomas and odontogenic keratocysts. British Journal of Oral and Maxillofacial Surgery 2004;42:381-90.

8. Jing $\mathrm{W}$ et al. Odontogenic Tumors: A retrospective study of 1642 cases in a chinese population. Int $J$ Oral and Maxillofac Surg 2007;36:20-25.

9. Ghali G.E., Connor M. Scott. Surgical management of the odontogenic keratocyst. Oral Maxillofacial SurgClin N Am 2003;15:383-92.

10. Schmidt Brian $L$. The use of liquid nitrogen cryotherapy in the management of the odontogenic keratocyst. Oral Maxillofacial Surg Clin N Am 2003;15:393-405. 